Aufsatz

Hannes Adomeit*

\title{
Bilanz der deutschen Russlandpolitik seit 1990
}

https://doi.org/10.1515/sirius-2020-3004

Zusammenfassung: Dieser Artikel untersucht, wie es dazu kommen konnte, dass sich all die Hoffnungen und Erwartungen, die sich 1990 mit dem in Moskau unterzeichneten Vertrag über die abschließende Regelung in Bezug auf Deutschland und der Charta von Paris für ein neues Europa verbanden, zerschlagen haben und warum sowohl die Vision einer deutsch-russischen „strategischen Partnerschaft" als auch ihre beabsichtigte Konkretisierung durch eine „Modernisierungspartnerschaft“ gescheitert sind. Der Artikel sieht die Erklärung für diese Entwicklung in der russischen Innenpolitik. Die russische Machtelite konnte auf die Angebote Deutschlands (und der EU) auf eine enge „Verflechtung“ und umfassende Zusammenarbeit nicht eingehen, weil diese ihren Wahrnehmungen zufolge den Machterhalt im eigenen Land und in der von ihr deklarierten Einflusssphäre in Mittel- und Osteuropa gefährdet hätte.

Schlüsselbegriffe: Deutschland, Russlandpolitik, europäische Sicherheit, Russland

\begin{abstract}
This article deals with the question as to why all the hopes and expectations associated with the Moscow Treaty on the Final Settlement with Respect to Germany and the Paris Charter for a New Europe, signed in 1990, have been shattered. It also asks why the vision of a German-Russian 'strategic partnership' and its corollary, the 'modernization partnership', have failed to come to fruition. The analysis sees the explanation for this development in Russian domestic politics. The Russian power elite considered it to be too risky to respond to the German (and EU) offers of integration (,Vernetzung“) and comprehensive cooperation because, according to its perceptions, this would have undermined its legitimacy internally and in the self-declared sphere of influence in Central and Eastern Europe.
\end{abstract}

Keywords: Germany, policy towards Russia, European security, Russia

*Kontakt: Dr. Hannes Adomeit: Senior Fellow, Institut für Sicherheitspolitik an der Universität Kiel (ISPK); E-Mail: hannes.adomeit@t-online.de

\section{Einleitung}

Die Politik gegenüber Russland nimmt einen hohen Stellenwert in der deutschen Außen- und Sicherheitspolitik ein. Gute Beziehungen zu Moskau gelten als wichtig für die Sicherung von Frieden und Zusammenarbeit in Europa. Der vorliegende Aufsatz befasst sich mit der Frage, wie sich die deutsch-russischen Beziehungen in den letzten drei Jahrzehnten entwickelt haben. Dabei wird insbesondere gefragt, wie es dazu kommen konnte, dass sich all die Hoffnungen und Erwartungen, die sich 1990 mit dem in Moskau unterzeichneten Vertrag über die abschließende Regelung in Bezug auf Deutschland und der Charta von Paris für ein neues Europa verbanden, zerschlagen haben. Ist die Zerrüttung des Verhältnisses zwischen Berlin und Moskau - wie manche Beobachter vermuten - eine Nebenwirkung des Abgleitens und Absturzes der russisch-amerikanischen Beziehungen oder gibt es dafür andere Gründe? Wie zutreffend ist das Narrativ, wonach Russland ganz im Einklang mit den Prinzipien der Charta von Paris dem Westen, einschließlich Deutschlands, die Hand zur umfassenden Zusammenarbeit entgegengestreckt habe, diese aber schnöde zurückgewiesen worden sei? Wie wichtig für die Zerrüttung der Beziehungen war die Osterweiterung der NATO? Und schließlich: Welche Möglichkeiten gibt es, eine grundsätzliche und nachhaltige Verbesserung des deutsch-russischen Verhältnisses zu bewerkstelligen, und böte dafür eine Rückkehr zur Ost- und Entspannungspolitik der 1970er-Jahre eine Grundlage? Dies sind die Kernfragen der nachfolgenden Untersuchung.

\section{Konzeptionelle Grundlagen der deutschen Russlandpolitik}

Deutschland hat Russland sowohl unter Präsident Boris Jelzin als auch unter Vladimir Putin fortlaufend Angebote einer engen Partnerschaft und umfassenden Zusammenarbeit gemacht. Die konzeptionelle Grundlage dafür war die Anschauung, dass Russland ein Land sei, das politisch, ökonomisch und kulturell zu Europa gehöre, und dass auch der geografisch zu Asien gehörende Teil „europäisch“ geprägt sei. Der Zusammenbruch des Sowjetsystems 
habe die Voraussetzungen dafür geschaffen, Russland fest in Europa zu verankern, es in Europa zu ,integrieren“ und „einzubinden“. Dies sollte entsprechend der in der Charta für ein neues Europa festgehaltenen Werte erfolgen. Deutschland war bereit, sich dafür zu engagieren und mitzuhelfen, Demokratie, Marktwirtschaft mit fairem Wettbewerb, einen Rechtsstaat und eine aktive Zivilgesellschaft aufzubauen. Dies würde auch die notwendigen Bedingungen für eine Abkehr des neuen Russlands von neoimperialen und „Großmacht“-Ambitionen in der Außenpolitik schaffen. Insofern gab es im deutschen Ansatz gegenüber Russland keine Trennung zwischen Werten und Interessen: Die Entwicklung Russlands zu einer friedlichen, an europäischen Werten orientierten Macht wurde als im ureigensten Interesse Deutschlands betrachtet.

Der Integrationsprozess sollte gemäß Axiomen wie „Wandel durch Handel“, „Wandel durch Annäherung“ und „Verflechtung“ vonstattengehen: Durch eine Ausweitung und Vertiefung wirtschaftlicher und gesellschaftlicher Kontakte durch „transnationale Netzwerke“ würden „Spillover"-Effekte geschaffen, die von den ökonomischen und gesellschaftlichen Ebenen auf die „höhere“ Ebenen des Regierungssystems, der Innenpolitik und der Außenpolitik übergriffen.

Eingebettet waren derartige Vorstellungen in die Postulate der Transformationstheorie. Dieser zufolge verliefen Prozesse des Übergangs von autoritären und zentralistischen Systemelementen sowjetischen Typs $\mathrm{zu}$ westlichen demokratischen, pluralistischen, marktwirtschaftlichen, rechtsstaatlichen und zivilgesellschaftlichen Strukturen - Transformationsprozesse also - in allen postkommunistischen Ländern, einschließlich Russlands, mehr oder weniger gleich. Nach einer Phase der Auflösung politischer und ökonomischer Strukturen und des wirtschaftlichen Absturzes würden neue, effiziente Institutionen geschaffen und die Wirtschaft käme wieder voran. In Staaten wie Polen, Tschechien oder Ungarn oder auch Estland, Lettland und Litauen, die erst im oder nach dem Zweiten Weltkrieg der Sowjetherrschaft unterworfen worden seien, hätten die Transformationsprozesse relativ schnell abgeschlossen werden können. Wegen des zaristischen und sowjetischen Erbes dauerten diese in Russland länger, aber prinzipiell sei der Weg auch für dieses Land vorausbestimmt und unumkehrbar.

Derartige Anschauungen können im Rückblick auf die damaligen Bedingungen nicht als unangemessen betrachtet werden. Das russische Feld, auf dem die Transformationsprozesse stattfinden sollten, war fruchtbar. Die in der Charta von Paris formulierten Werte für ein neues Europa entsprachen den Paradigmen des in der Gorbačëv-Ära entwickelten und anfangs von Jelzin weiter geführten Konzep- ten der Demokratisierung in der Innenpolitik, des Umbaus der Wirtschaft zur Marktwirtschaft, größerer Transparenz im Verhältnis zur Gesellschaft und des Herunterfahrens der Rüstungsanstrengungen auf ein den Ressourcen des Landes und internationalen Gegebenheiten angemessenes Maß. In der Außenpolitik galten weiterhin die Prinzipien des „Neuen Politischen Denkens“, des „Gemeinsamen Hauses Europa“ und der Anerkennung der „Freiheit der Wahl“ für die Nachbarstaaten, ihre innere Verfassung und außenpolitische Orientierung eigenständig zu entwickeln. ${ }^{1}$ Die Bedrohungen der nationalen Sicherheit Russlands hätten im Wesentlichen keinen militärischen Charakter, sondern seien hauptsächlich innerer Natur und gründeten auf den ernsten wirtschaftlichen, sozialen und ökologischen Problemen des Landes. Die Außenpolitik sollte dazu dienen, dringend gebotene innere Reformen zu begünstigen. ${ }^{2}$

Warum ist die Saat eines europäischen Werten verpflichteten Russlands und der Abkehr von einer eng verstandenen „Großmacht“-Politik nicht aufgegangen? Die Antwort ist in der russischen Innenpolitik zu finden.

\section{Das Scheitern der deutschen Russlandpolitik}

Schon 1992 geriet die europäische und euro-atlantische Orientierung Kozyrevs massiv unter Druck. Diese wurde in der Jelzin-Ära nach und nach ausgehöhlt und unter Vladimir Putin nach 1999 verlassen, wobei die Endphase der Amtszeit Dmitrij Medvedevs als Präsident im Herbst 2011 und der Beginn der dritten Amtszeit Putins im Frühjahr 2012 eine wichtige Zäsur darstellen. Die Entwicklung des russischen Regierungssystems folgte trotz wiederholter offizieller Bekenntnisse nicht den im Westen postulierten Transformationsprozessen hin zu Demokratie, Marktwirtschaft, Rechtsstaat und Zivilgesellschaft europäischen oder westlichen Zuschnitts, sondern bestand konzeptionell in der Rückkehr zum alten Denken und praktisch im Aufbau eines Systems sui generis, dem System Putin. In

1 Die entsprechenden russischen Begriffe für die konzeptionelle Neuorientierung unter Gorbačëv sind wie folgt: Demokratisierung demokratizacija; Umbau - perestrojka, Offenheit und Transparenz glasnost‘; Vernünftiges Maß, wörtlich: Hinlänglichkeit (von Rüstungsanstrengungen) - razumnaja dostatočnost'; Neues Politisches Denken - novoe političsekoe myšlenie; Gemeinsames Haus Europa Evropa, naš obščij dom; und Freiheit der Wahl - svoboda vybora.

2 Die Inhalte des „Neuen Politischen Denkens“ und ihre Bedeutung ausführlich bei Adomeit/Bomsdorf 1990. 
diesem System wurde der ideologische Klebstoff des Marxismus-Leninismus zwar entfernt, wesentliche Strukturelemente des Sowjetsystems wurden aber übernommen: die Rückkehr des Kremls zum Denken in machtpolitischen Kategorien, in Einflusssphären, Kräftekorrelationen und Machtvakua und die Rückkehr zu einem Bild der euro-atlantischen Welt, die Russland - mit der NATO als Speerspitze - prinzipiell feindlich gegenüber eingestellt sei. Dieses Narrativ konnte letzten Endes zu nichts anderem als zu der Erkenntnis führen, dass die Hoffnungen und Erwartungen Deutschlands auf die Herstellung einer „strategischen Partnerschaft" mit Russland illusorisch waren. Diese Entwicklung kann hier nicht Schritt für Schritt und Jahr für Jahr rekonstruiert werden. Nur die wichtigsten Trendlinien und Meilensteine des Wegs können aufgezeigt werden.

\section{Grundstrukturen des Verhältnisses}

Die Bedingungen für eine tiefgreifende und umfassende Neuordnung des Verhältnisses zwischen dem wiedervereinigten Deutschland und dem neuen Russland nach dem Zusammenbruch der Sowjetunion waren günstig. Die Außenpolitik der Russischen Föderation ab ihrer Souveränitätserklärung (Juni 1990) mit Andrej Kozyrev an der Spitze des entsprechenden Ministeriums (ab Oktober 1990) beruhte auf dem in der Gorbačëv-Ära entwickelten Konzept des Neuen Politischen Denkens. Dazu gehörte der Abbau des Feindbilds Westen, der immer und ewig darauf aus sei, Russland in die Knie zu zwingen. Russland, wie Präsident Boris Jelzin Anfang 1992 vor den Vereinten Nationen ausführte, betrachte die westlichen Länder nicht mehr als Gegner, sondern als „Verbündete“; ${ }^{3}$ und folgerichtig erklärte er die Mitgliedschaft des Landes in der Nato zu einem langfristigen Ziel. ${ }^{4}$ Das neue Russland war bereit, in oder mit den bestehenden internationalen politischen und wirtschaftlichen Organisationen wie den Vereinten Nationen, der EU, dem Internationalen Währungsfonds, GATT und der G7 zusammenzuarbeiten und Mitglied in der WTO zu werden. Das globale Überengagement der Sowjetunion wurde weiter abgebaut, Russland rückte von dem traditionellen sowjetischen Ziel militärstrategischer Parität mit den USA ab, und es trieb den Abzug russischer Truppen aus Deutschland energisch voran.

3 Diplomatičeskij vestnik, 29. Februar 1992.

4 Diplomatičeskij vestnik, 15. Januar 1992.
In der Innenpolitik des neuen Russlands stand das Bemühen im Vordergrund, eine Demokratie ohne einschränkende Adjektive mit einer funktionierenden Gewaltenteilung, rechtsstaatlichen Verhältnissen und einer aktiven Zivilgesellschaft aufzubauen. In der Wirtschaft sollte die Abhängigkeit von Rohstoffen überwunden, die Vorrangstellung des militärisch-industriellen Komplexes beseitigt, der Verfilzung von Staatsbürokratie mit der organisierten Kriminalität ein Ende gesetzt und freiem Unternehmertum im Rahmen einer Marktwirtschaft mit fairem Wettbewerb Raum gegeben werden. Der russischen Innenpolitik wurde Vorrang vor der Außenpolitik mit dem Argument gegeben, dass politische, wirtschaftliche, wissenschaftlich-technologische, ökologische und informationstechnische Faktoren in der internationalen Politik zunehmend an Bedeutung gewönnen und die Bedrohungen der nationalen Sicherheit Russlands hauptsächlich in ernsten wirtschaftlichen, sozialen und ökologischen Problemen des Landes zu finden seien. Sogar die nukleare Abschreckungsfähigkeit wurde in diesen Zusammenhang gestellt: Der nukleare Schirm und die Neuordnung der Beziehungen zur Außenwelt machten es Russland leichter, „die Ressourcen des Staates und der Gesellschaft neu zugunsten einer prioritären Lösung der akuten inneren Probleme zu verteilen." ${ }^{\text {5 }}$

Im Rahmen der europäischen und transatlantischen Institutionen, wie EU und NATO, trug Deutschland dazu bei, den Erfolg dieser Neuordnung zu sichern. Im Zweiplus-Vier-Vertrag hatte es sich verpflichtet, auf Herstellung und Besitz von und auf Verfügungsgewalt über atomare, biologische und chemische Waffen zu verzichten und die Streitkräfte des vereinten Deutschlands innerhalb von drei bis vier Jahren auf eine Personalstärke von 370.000 Mann (Land-, Luft- und Seestreitkräfte) zu reduzieren. Um nationale Alleingänge auszuschließen, blieben die deutschen Truppen fest in die politischen und militärischen Strukturen des atlantischen Bündnisses eingebunden. Dem Vertrag über konventionelle Streitkräfte in Europa (KSE-Vertrag) entsprechend wurden umfangreiche Reduzierungen der Anzahl schwerer konventioneller Waffensysteme (Kampfpanzer, gepanzerte Kampffahrzeuge, Artilleriesysteme, Kampfflugzeuge sowie Angriffshubschrauber) vorgenommen und durch Meldungen der Bestände und Vor-Ort-Inspektionen Berechenbarkeit und gegenseitiges Vertrauen erhöht. Probleme wie die ungelöste Beutekunst-

5 Koncepcija nacional'noj bezopasnosti Rossijskoj Federacii [Konzept der nationalen Sicherheit der Russländischen Föderation], Rossijskaja gazeta, 26.12.1997. Zu dem Zeitpunkt, in dem das Konzept veröffentlicht wurde, war es allerdings schon in der außenpolitischen Praxis überholt. 
frage oder die Rehabilitierung der in Russland lebenden deutschen Minderheit belasteten das deutsch-russische Verhältnis nicht wirklich.

Deutschland machte dem Russland unter Boris Jelzin Angebote einer engen Partnerschaft und umfassender Zusammenarbeit. Die praktische Anwendung dieses Ansatzes erfolgte im Rahmen des am 24. Juni 1994 beschlossenen EU-Russland-Partnerschafts- und Kooperationsabkommen (PKA), das am 1. Dezember 1997 in Kraft trat. Die beste Zusammenfassung sowohl der theoretischen Fundierung als auch der zu ergreifenden praktischen Maßnahmen für die Entwicklung der deutsch-russischen Beziehungen findet sich jedoch in der Gemeinsamen Strategie der Europäischen Union gegenüber Russland, die am 4. Juni 1999 auf der EU-Gipfelkonferenz in Köln verabschiedet wurde. $^{6}$

\section{Die deutsch-russische „strategische Partnerschaft“}

Das Dokument trägt unverkennbar die deutsche Handschrift. Deutschland hatte zu diesem Zeitpunkt den Vorsitz im Europäischen Rat inne und sorgte dafür, dass die Russlandpolitik der EU vor dem Beitritt der mittelosteuropäischen Staaten zur EU im Wesentlichen deutschen Vorstellungen und Vorgaben folgte.

So heißt es in der Einleitung zu diesem Dokument, dass der Europäische Rat die Gemeinsame Strategie zur „Festigung [sic] der strategischen Partnerschaft zwischen der EU und Russland am Beginn eines neuen Jahrhunderts“ annehme und dabei anerkenne, dass die Zukunft Russlands „,wesentlicher Bestandteil der Zukunft des Kontinents und für die EU von strategischem Interesse ist. Das Angebot einer gefestigten Partnerschaft auf der Grundlage gemeinsamer demokratischer Werte wird Russland bei der Behauptung seiner europäischen Identität unterstützen und allen Menschen auf dem Kontinent neue Möglichkeiten eröffnen. Durch die Erweiterung der EU werden diese Vorteile und Möglichkeiten sich noch ausweiten.“

Dabei standen die folgenden Aktionsbereiche im Mittelpunkt bei der Ausführung der Gemeinsamen Strategie der EU: ${ }^{7}$

6 Wortlaut des Dokuments zitiert nach: https://www.europarl.europa. eu/summits/kol2_de.htms. Die „Europäische Union“ ist hier durchweg durch „EU“ ersetzt worden.

7 Die Liste der konkreten Angebote ist lang. Nur einige von diesen werden hier beispielhaft und teilweise verkürzt genannt.
„Festigung des Rechtsstaats und Stärkung der staatlichen Institutionen

- $\quad$ durch Unterstützung und Förderung der notwendigen institutionellen Reformen zur Schaffung einer modernen und effizienten Verwaltung im Rahmen von Russlands Exekutive, Legislative und Judikative auf föderaler, regionaler und lokaler Ebene, insbesondere durch den Ausbau der Kapazität einer unabhängigen Justiz, der öffentlichen Verwaltung und rechenschaftspflichtiger Strukturen zur Strafverfolgung, durch die Förderung der Kontakte zwischen den Justizbehörden und den Strafverfolgungsbehörden der Mitgliedstaaten der EU und Russlands.

Stärkung der Bürgergesellschaft

- durch Förderung der Kontakte zwischen russischen Politikern und Politikern aus der EU auf föderaler, regionaler und lokaler Ebene einschließlich Versammlungen auf allen Ebenen;

- $\quad$ durch die Ausweitung von Stipendien und Programmen für Kontakte zwischen Studenten;

- $\quad$ durch Unterstützung unabhängiger Nichtregierungsorganisationen;

- $\quad$ durch Unterstützung der Freiheit der Medien.

Konsolidierung des Wirtschaftsreformprozesses

- $\quad$ durch die Unterstützung bei der Schaffung und Nutzung eines transparenten und stabilen rechtlichen und ordnungspolitischen Rahmens, mit dem die Wirtschaftstätigkeit und verstärkte in- und ausländische Investitionen gefördert werden sollen:

- $\quad$ durch die Förderung glaubwürdiger Reformen des Bankensektors mit fairen Bedingungen für Auslandsbanken und einer wirksamen Koordinierung zwischen den Gebern unter umfassender Beteiligung der EU;

- $\quad$ durch Unterstützung weiterer struktureller, wirtschaftlicher und administrativer Reformen, einschließlich einer weiteren Privatisierung, der Unternehmensumstrukturierung und der Zunahme der KMU in Russland.

Integration Russlands in einen größeren Raum der wirtschaftlichen Zusammenarbeit

- $\quad$ durch die Unterstützung der weiteren Integration Russlands in das multilaterale Handelssystem sowie der russischen Bemühungen um die Erfüllung der Bedingungen für den WTOBeitritt, einschließlich der rechtlichen und institutionellen Reformen;

- $\quad$ durch Prüfung der Frage, wie die erforderlichen Voraussetzungen für die künftige Errichtung einer Freihandelszone zwischen der EU und Russland geschaffen werden können;

- durch Ausarbeitung eines Plans gemeinsamer Aktionen mit Russland zur Bekämpfung der organisierten Kriminalität, einschließlich der Korruption, der Geldwäsche, des Drogenund Menschenhandels und der illegalen Einwanderung."

Die in der Gemeinsamen Strategie aufgezeichneten Vorstellungen über die Entwicklung der Beziehungen zu Russland sind eindeutig: Die Transformationsprozesse sollten auf breiter Basis und primär „von unten nach oben“ vonstattengehen und horizontal mittels enger 
gesellschaftlicher und wirtschaftlicher Verflechtung, die von staatlicher Seite mit politischem Nachdruck und finanziell gefördert werden sollte. Dies erfolgte und führte zu beachtlichen Ergebnissen.

\section{Ergebnisse des deutschen Ansatzes}

In den eineinhalb Jahrzehnten nach Annahme der Gemeinsamen Strategie entwickelte sich zwischen Deutschland und Russland auf politischer, wirtschaftlicher und gesellschaftlicher Ebene eine Breite und Dichte von Kontakten, die von keinem anderen Land der EU erreicht wurde. Jährlich wurden deutsch-russische Regierungskonsultationen abgehalten, an denen jeweils die Vertreter der für die Beziehungen wichtigsten Ministerien und Ämter teilnahmen. Neben der Botschaft in Moskau unterhielt Deutschland vier Konsulate: in St. Petersburg, Kaliningrad, Ekaterinburg und Novosibirsk. Beamte und Offiziere aus den Ministerien beider Seiten nahmen an vielzähligen bilateralen Konferenzen und Gesprächsforen teil - so beispielsweise an dem unter der Schirmherrschaft des jeweils amtierenden deutschen Bundeskanzlers und des jeweils amtierenden russischen Präsidenten stehenden Petersburger Dialogs und dem von der Stiftung Wissenschaft und Politik (SWP) jährlich in Zusammenarbeit mit dem Bundesverteidigungsministerium durchgeführten Dialog mit russischen Generälen.

Im Bereich der Wirtschaftskooperation waren unter anderem die vom Bundesministerium für Arbeit und Wirtschaft koordinierte Strategie-Arbeitsgruppe, der Ost-Ausschuss der deutschen Wirtschaft sowie deutsche und russische Industrievertreter im Rahmen von Rundtischgesprächen tätig. Die Wirtschaftsbeziehungen entwickelten sich auf der Basis einer fundamentalen Komplementarität: Russland war für Deutschland als Lieferant von Rohstoffen und Energieträgern, insbesondere von Erdöl und Erdgas, wichtig und Deutschland für Russland als Anbieter von Erzeugnissen der verarbeitenden Industrie zur Umstrukturierung und Modernisierung der russischen Wirtschaft. Deutschland bezog rund ein Drittel seines importierten Erdöls aus Russland, was knapp 20 \% der russischen Erdölexporte ausmachte. Rund $40 \%$ der deutschen Erdgaseinfuhren stammten aus Russland, was ca. $25 \%$ des russischen Erdgasexports entsprach.

Russland führte mit Abstand die Liste der Empfängerliste für Hermesbürgschaften an, die jeweils jährlich mehrere Milliarden Euro ausmachten. Das trug dazu bei, dass das deutsch-russische Handelsvolumen von 13,5 Mil- liarden Euro im Jahre 2000 auf rund 80 Milliarden Euro 2013 anstieg. Bis zu diesem Jahr summierten sich die deutschen Investitionen in Russland auf rund 20 Milliarden Euro. Mehr als 6.400 deutsche Firmen verfügten über Niederlassungen in Russland. Die deutsche Präsenz in Russland ging aber weit über Firmenrepräsentanzen hinaus und umfasste eine Vielzahl von Verbänden und Stiftungen.

Für den Aufbau von Demokratie und Marktwirtschaft erhielt Russland im Rahmen des 1994 eingerichteten „Transform“-Programms der Bundesregierung Finanzmittel in Höhe von rund 350 Millionen Mark und 1,2 Milliarden Euro aus Mitteln des TACIS-Programms der EU. Deutschland engagierte sich bei Umweltproblemen, darunter bei der Vernichtung von Chemiewaffen und zusammen mit den USA bei der Entsorgung der atomgetriebenen U-Boote der russischen Nordmeerflotte.

Zudem umfasste das deutsche Engagement vielfältige gesellschaftliche Beziehungen. Russland wurde zum Hauptadressat der deutschen auswärtigen Kulturpolitik. Das Goethe-Institut eröffnete neben dem Hauptsitz in Moskau und der Vertretung in Petersburg ein weiteres Büro in Novosibirsk. Mehr als 80 Städte pflegten Partnerschaften mit Städten in Russland, wobei nicht nur Großstädte wie Berlin und Moskau oder Hamburg und St. Petersburg kooperierten, sondern auch in den Regionen enge Kooperationsformen entstanden. Der Wissenschaftsaustausch umfasste mehr als 500 Hochschulpartnerschaften. Mehr als 300 deutsche Nichtregierungsorganisationen engagierten sich für gesellschaftliche, kulturelle und letzten Endes auch politische Zusammenarbeit, darunter auch das Deutsch-Russische Forum und der Deutsch-Russische Austausch sowie die Stiftungen der politischen Parteien - die Konrad-Adenauer-, Friedrich-Ebert-, Friedrich-Naumann- und Heinrich-Böll-Stiftung. Eine Vielzahl von Konferenzen und Workshops wurde von deutschen Institutionen wie der Deutschen Gesellschaft für Auswärtige Politik (DGAP), der Stiftung Wissenschaft und Politik (SWP) und der Deutschen Gesellschaft für Osteuropakunde (DGO) mit sowohl offiziellen als auch regierungsunabhängigen russischen Teilnehmern abgehalten. Regelmäßig tagende Gesprächsforen wurden eingerichtet, so beispielsweise die von der Hessischen Stiftung für Friedens- und Konfliktforschung (HSFK) mit der Moskauer Vertretung der Friedrich-Ebert-Stiftung jährlich veranstalteten Schlangenbader Gespräche.

So beeindruckend wie diese Bestandsaufnahme enger wirtschaftlicher und gesellschaftlicher deutsch-russischer Verflechtungen und Verbindungen vom Zusammenbruch der Sowjetunion bis Russlands Annexion der Krim auch sein mag, gibt diese dennoch keinen Aufschluss darüber, was nicht erreicht wurde. Zudem muten die erzielten 
Ergebnisse sogar in dem für Russland wichtigen Bereich der Wirtschaft im internationalen Vergleich und insbesondere mit den mittelosteuropäischen Ländern eher bescheiden an. Dies kann anhand einiger Zahlen veranschaulicht werden: Im Jahr 2013 betrug der Anteil Russlands an den gesamten deutschen Exporten nur 3,2\%. Das Land belegte damit nur den 11. Platz auf der Liste der deutschen Exportdestinationen. Bei den ausländischen Direktinvestitionen (FDI) nach Russland rangierte Deutschland in jenem Jahr lediglich auf Platz 6 - hinter Zypern, den Niederlanden, Luxemburg, China und Großbritannien. Die 20 Milliarden Euro kumulierter deutscher FDI im Jahre 2013 (siehe oben) stellten nur einen geringen Teil der deutschen Direktinvestitionen im Ausland in Höhe von 369 Milliarden Euro dar. Der Nettotransfer von Kapital von Deutschland nach Russland in diesem Jahr war negativ und belief sich auf 113 Millionen Euro, Russland investierte also mehr in Deutschland als umgekehrt. Im Vergleich dazu lag der deutsche Kapital-Nettotransfer in die Tschechische Republik mit 3,2 Mrd. Euro und nach Polen mit 2,1 Mrd. Euro im Plusbereich. ${ }^{8}$

Aufschluss geben die Ergebnisse - soweit diese positiv waren - auch darüber nicht, warum die Verbindungen und Verflechtungen keinen Spillover in die Grundstrukturen des Regierungssystems und die russische Außenpolitik bewirken konnten. Um die Erklärung dafür zu finden, ist es notwendig, auf die Entwicklungen in Russland seit Jelzin einzugehen.

\section{Rückkehr zum alten Denken in Moskau}

Schon wenige Monate nach dem Zusammenbruch der Sowjetunion gerieten das in der Gorbačëv-Ära entwickelte Konzept des Neuen Politischen Denkens und die euroatlantische Orientierung des russischen Außenministeriums innenpolitisch unter Beschuss. Am 15. Dezember 1992 kündigte Außenminister Andrej Kozyrev auf der Ratskonferenz der KSZE-Staaten in Stockholm einige „Korrekturen“ seiner Politik an. Er griff angebliche Pläne des Westens für eine „militärische Präsenz in den Baltischen Staaten und anderen Gebieten der ehemaligen UdSSR“ an und warnte, dass Russland seine Interessen auf dem gesamten Gebiet der ehemaligen Sowjetunion verteidigen und dabei „alle verfügbaren Mittel, einschließlich militärischer und ökonomischer Mittel“ einsetzen werde. „Wir werden [zudem]

8 Einzelheiten und Quellenangaben bei Adomeit 2016. nachdrücklich darauf bestehen, dass ehemalige Sowjetrepubliken [...] einer neuen Föderation oder Konföderation beitreten." Bezüglich der Konflikte auf dem Balkan kritisierte er die „Einmischung“ des Westens in die inneren Angelegenheiten Jugoslawiens, sicherte Serbiens Präsidenten Slobodan Miloševićs die Unterstützung des ,großen Russlands“ (velikoj Rossii) zu und forderte, die vom UN-Sicherheitsrat gegen Serbien verfügten Wirtschaftssanktionen aufzuheben. ${ }^{9}$ Eine Stunde nach der Ankündigung der „Korrekturen“ kehrte Kozyrev zum Konferenzraum zurück und erklärte den konsternierten Außenministerkollegen, die von ihm skizzierte Richtungsänderung sei lediglich eine Warnung gewesen, was passieren würde, wenn sich die gegen seinen Kurs agierenden national-patriotischen Kräfte in Russland durchsetzen würden.

Diese setzten sich tatsächlich durch. Kozyrev wurde vorgeworfen, er stufe Russland zu einem „Lakaien“ und „Handlanger“ der Vereinigten Staaten herab und betreibe den „Ausverkauf“ russischer Interessen. Als besonders „schändlich“wurde die Vernachlässigung der „Rechte“ russischer Minderheiten im „Nahen Ausland“ gebrandmarkt. So wurde nach scharfen Auseinandersetzungen und mehreren Entwürfen im April 1993 ein neues außenpolitisches Konzept von einer neu gegründeten ressortübergreifenden Kommission des Nationalen Sicherheitsrats verabschiedet, in welchem die Reintegration der neuen unabhängigen Staaten im Rahmen der GUS, die Schaffung eines ,einheitlichen militärstrategischen Raums“ und der Schutz der russischen Minderheiten im postsowjetischen Raum Vorrang gegeben wurden..$^{10}$ Entsprechend wandte sich Jelzin an ,autoritative internationale Organisationen, die Vereinten Nationen eingeschlossen, Russland [in diesem Raum] Sonderrechte als Garantie[macht] für Frieden und Stabilität" einzuräumen. ${ }^{11}$ Wie ein roter Faden zieht sich diese russische Version der Monroe Doktrin von der Jelzin-Ära bis zur Gegenwart, mit dem Unterschied allerdings, dass Putin, wie die Militärinterventionen in Georgien, auf der Krim und in der Ostukraine bewiesen, keine internationale Organisation um Autorisierung bittet, sondern ein unilaterales Recht auf Intervention in Anspruch nimmt.

Die Abkehr vom Neuen Denken und der euroatlantischen Orientierung wurde auch in einer Studie des russischen Auslandsgeheimdienstes (SVR) unter Leitung von Evgenij Primakov deutlich. In dem im November 1993 vorgestellten Dokument wurde die NATO wieder als die „größte militärische Gruppierung der Welt, die ein

9 Andrej Kozyrev na sessii Soveta SBSE, Kommersant, 16.12.1992, https://www.kommersant.ru/doc/33555.

10 Sovet bezopasnosti 1993.

11 ITAR-TASS (russ.), 1.3.1993 (Hervorhebung nicht im Original). 
enormes Offensivpotential besitzt“, bezeichnet. Der westlichen Militärallianz wurde unterstellt, „den Stereotypen des Blockdenkens“ verhaftet zu sein, und sie wurde eindringlich davor gewarnt, sich nach Osten auszudehnen. ${ }^{12}$ Jelzins Pressesprecher warnte sogar, dass das „Vordringen" der NATO in Gebiete in unmittelbarer Nähe zur russischen Grenze zu einer „militärpolitischen Destabilisierung der Region" führe. ${ }^{13}$

Schon in dieser frühen Zeitperiode kann man von der Herausbildung eines „patriotischen Konsens“ sprechen. ${ }^{14}$ Darin fanden sich Vertreter verschiedener antiwestlicher Strömungen und Kräfte, Anhänger von „Großmacht“-Visionen (deržavniki), „Eurasier“, „Eurasisten“, Nationalisten, Chauvinisten und Kommunisten zusammen. Parteipolitisch wurden sie unter anderem durch die weder liberale noch demokratische Liberal-Demokratische Partei Russlands (LDPR) Vladimir Žirinovskijs, die der Sowjetunion und dem Sowjetimperium nachtrauernde Kommunistische Partei der Russischen Föderation (KPRF) Gennadij Zjuganovs und die mit ihr verbündete Agrarpartei repräsentiert.

Bei den Wahlen zur Duma vom Dezember 1993 gingen diese Parteien klar als Gewinner hervor. Die LDPR erzielte mit 22,92 Prozent der abgegebenen Stimmen das beste Ergebnis, die KPRF erhielt 12,4 Prozent und die Agrarier bekamen 7,99 Prozent. Die „rot-braunen“ Gegner von Demokratie und euroatlantischer Orientierung erhielten also insgesamt 43,29 Prozent der Stimmen. Die reformistisch-demokratischen Parteien wie Russlands Wahl und Jabloko kamen offiziell nur auf 15,5 Prozent respektive 7,86 Prozent.

Die Ergebnisse erinnerten an die Verhältnisse und das Schicksal der Weimarer Republik: die von Nationalisten, Faschisten und Kommunisten betriebene Zerstörung der Demokratie und Westorientierung mit all ihrer revisionistischen Agitation und Propaganda, der zufolge mit dem westlichen Ausland zusammenarbeitende Kräfte für den Zusammenbruch des Landes verantwortlich waren. Sie warfen - insbesondere unter deutschen Russlandexperten - schon zu diesem frühen Zeitpunkt die Frage auf,

12 Der 30 Seiten umfassende Text der Studie wurde an Journalisten auf einer Pressekonferenz in Moskau verteilt und vollständig oder in Auszügen in allen großen nationalen Zeitungen veröffentlicht; Služba vneshnej razvedki (1993): Perspektivy rasširenija NATO i interesy Rossii. Doklad služby vneshnei razvedki“, Izvestija, 26.11.1993. Primakov löste Kozyrev im Januar 2006 in seinem Amt als Außenminister ab und war von September 1998 bis Mai 1999 Premierminister.

13 ITAR-TASS, 5.1.1994.

14 Zum „patriotischen Konsens“ siehe Arbatov, Aleksej (1997): Vnešnepolitičeskij konsens v Rossii, Nezavisimaja gazeta, 14.3.1997 sowie Alexandrova 1997 und Simon 1997. wie Russland zu verstehen sei: als sicherheitspolitischer „Partner“ oder als „Risikofaktor“? 15

Einer der wichtigsten Meilensteine und Beweisstück für die von Kozyrev befürchtete „Korrektur“ der außenpolitischen Orientierung Russlands ist die Haltung Jelzins und der nun wieder einflussreichen Vertreter neoimperialen und machtpolitischen Denkens zu den Balkankriegen.

Nach dem Zusammenbruch Jugoslawiens stellten sie sich auf die Seite Serbiens unter Slobodan Milošević. Dabei verwiesen sie auch auf die historisch begründete „traditionelle Verbundenheit“ Russlands mit diesem slawischen und christlich-orthodoxen Land. Nach dem durch bosnisch-serbische Artilleriegeschosse verursachten Massaker auf dem Marktplatz von Sarajewo im August 1995 und den daraufhin von der NATO Anfang September durchgeführten Luftangriffen gegen serbische Stellungen ließ sich Jelzin zu dem Vorwurf verleiten, die NATO betreibe „Genozid an den Serben“ und „beschwöre die Flamme eines neuen Weltkriegs über Europa“ herauf. Die Duma bezeichnete die Vertreibung von angeblich 600.000 Serben aus der Kraijna als „größte humanitäre Katastrophe in Europa seit dem Zweiten Weltkrieg“. Der Aufschrei der Empörung und die verbalen Ausfälle als Reaktion auf den Luftkrieg der Nato gegen Jugoslawien im Jahre 1999 fielen noch schärfer aus. Jelzin bezeichnete die Angriffe der NATO als völkerrechtswidrige Aggression und kündigte „adäquate Maßnahmen“ gegen den Westen an, sollten russische Sicherheitsinteressen direkt berührt werden. Das emotional aufgeladene Wort vom „Genozid“ der NATO an den Serben wurde erneut bemüht, nunmehr von Außenminister Sergej Ivanov. Ein Sprecher des Verteidigungsministeriums kündigte an, die russischen Streitkräfte würden in erhöhte Alarmbereitschaft versetzt, und der Vorsitzende der Duma sprach davon, Jelzin habe ihm gegenüber erklärt, russische Atomsprengköpfe würden nunmehr wieder auf westliche Ziele gerichtet.

Politisch schwerwiegend und für die Entwicklung Russlands und die deutsch-russischen Beziehungen tragisch zu bewerten ist die Tatsache, dass sich der als Demokrat und pro-westlich gefeierte Jelzin, der den Putschisten des August 1991 mutig entgegentrat und mit Bundeskanzler Kohl in demonstrativer persönlicher Freundschaft und „Sauna-Diplomatie“ verbunden war, nicht nur nicht bemühte, den national-patriotischen Umtrieben Einhalt zu gebieten, sondern sich an ihre Spitze setzte.

Wie in der praktischen Politik auf dem Balkan offenbarte sich die Abkehr des Kremls von der euroatlantischen Orientierung auch in den konzeptionellen Ansätzen.

15 Adomeit 1994. 
Dies wurde am Übergang zum neuen Millennium - von der Jelzin-Ära zu Putin - in Russlands Antwort auf die Gemeinsame Strategie der EU deutlich, die Putin in seiner Eigenschaft als Premierminister der EU-,Troika“ auf der EU-Russland Gipfelkonferenz in Helsinki am 22. Oktober 1999 übergab. ${ }^{16}$

\section{Strategische Partnerschaft im russischen Verständnis}

Die russische „Strategie“ beinhaltete im Kern allerdings keine kooperative Aufnahme, sondern eine Zurückweisung des EU-Angebots. Der wichtigste Punkt dabei war die Abwehr jeglicher, von der Bundesregierung und der EU gehegten Vorstellung von Supranationalität und Integration in einen gemeinsamen europäischen Wirtschaftsund Rechtsraum. So erklärte Moskau, dass seine Strategie hauptsächlich dazu dienen solle, „Russlands nationale Interessen zu sichern und seine Rolle sowie sein Image in Europa und der Welt zu stärken“. Die Strategie solle der „objektiven Notwendigkeit dienen, eine multipolare Welt“ zu schaffen.

Das Dokument begründet die Zurückweisung der deutschen und EU-Vorstellungen (unausgesprochen) mit dem Argument, Russland sei eben nicht nur ein europäisches Land wie jedes andere, geschweige denn nur eine europäische Mittelmacht, sondern ,eine auf zwei Kontinenten angesiedelte Weltmacht“, ein „euro-asiatischer Staat“ und das „größte Land der GUS“. Aufgrund dieser Attribute, so war zu folgern, müsse „Russland frei sein, seine Innen- und Außenpolitik zu bestimmen und auszuführen, seinen Status und seine Vorteile als euro-asiatischer Staat und größtes Land der GUS [zu nutzen] und seine Unabhängigkeit [...] in internationalen Organisationen [zu bewahren]“.${ }^{17}$ Der Begriff der Integration wird

16 Strategija razvitija otnošenij Rossijskoj Federacii s Evropejskim Sojuzom po srednescročnuju perspektivu (2000-2010), http://www. ieras.ru/journal/journal1.2000/9.htm. Titel des Dokuments, übersetzt: „Strategie der Russischen Föderation gegenüber der Europäischen Union in mittelfristiger Perspektive (2000-2010). Darstellung und Analyse der Mittelfristigen Strategie Russlands bei Adomeit 2013, 130-132. Putin war am 9. August von Jelzin zum Ministerpräsidenten ernannt worden. Am 1. Oktober hatten russische Armeeeinheiten auf Befehl Putins die Grenze nach Tschetschenien überschritten. Die EU-,Troika“ bestand aus Ratspräsident, Hohem Vertreter der GASP und dem Kommissionspräsidenten.

17 In der Mittelfristigen Strategie wird noch ein eher geografisch bestimmter Begriff für die Charakterisierung der Andersartigkeit Russlands gebraucht, nämlich „euro-asiatisch“ (evroaziackij). In all den zwar verwandt, aber mit einer ganz anderen als der von der EU gewünschten Zielrichtung: Russland beabsichtige, „die positive Integrationserfahrung der EU mit Blickrichtung auf die Konsolidierung und Weiterentwicklung von Integrationsprozessen in der GUS zu nutzen“.

Diese Absichtserklärung fügte sich nahtlos in den Anspruch Moskaus auf den postsowjetischen Raum als russische Einflusssphäre ein. Dies ist nicht nur dem wiederholten Hinweis auf Russland als „euro-asiatischer Staat“ zu entnehmen, sondern auch der Erläuterung, dass die Partnerschaft mit der EU dazu beitragen solle, „Russlands Rolle als die führende Macht bei der Schaffung eines neuen Systems politischer und wirtschaftlicher zwischenstaatlicher Beziehungen auf dem Gebiet der GUS zu konsolidieren." Die Osterweiterung der EU hätte nicht nur „ambivalente Auswirkungen“ auf die Zusammenarbeit, sondern Moskau behielte sich „das Recht vor, [seine] Zustimmung zur Anwendung des [EU-Russland-] Partnerschafts- und Kooperationsabkommens" auf neue Beitrittsländer in Ostmitteleeuropa zu verweigern.

Die von Jelzin quasi offiziell auf dem G7-Gipfel in Birmingham im Mai 1998 gezogene „rote Linie“ entlang der Außengrenzen der ehemaligen Sowjetunion, welche die NATO nicht überschreiten dürfe, wird in der Mittelfristigen Strategie de facto auch für die EU gezogen: Russland werde sich ,jeglichen Anstrengungen, die ökonomische Integration in der GUS zu behindern, widersetzen." Es sei „gegen jedwede Sonderbeziehungen der EU mit einzelnen GUS-Ländern zum Schaden russischer Interessen“.

Was waren die Gründe für die Abkehr vom Neuen Denken Gorbačëvs und der euroatlantischen Orientierung Kozyrevs? Zum großen Teil lagen sie in den Interessen der Institutionen, welche die Größe Russlands mit militärischer Macht gleichzusetzen pflegen: das Militär, die Sicherheitsdienste und der militärisch-industrielle Komplex. Den Ministerien, Ämtern und Betrieben des immer noch weit gespannten, aber maroden Netzwerks politischer und wirtschaftlicher Macht war daran gelegen, die Grundlage für größere Mittelzuweisungen zu schaffen. Diesem Interesse war Entspannung mit dem Westen abträglich. Das Narrativ von der NATO als „offensivem“ Militärbündnis, das „immer näher an Russlands Grenzen“ heranrücke, passte dazu ebenso wie die Aufrechterhaltung einer über eine Million Mann umfassenden Massenarmee, die sich auf einen „großmaßstäblichen Krieg“ (krupnomasštabnaja vojna), im Klartext mit der NATO, vorbereiten müsse.

späteren Dokumenten, die sich auf die Zollunion, den EWR und die Eurasische Union beziehen, wird dagegen der eher ideologisch gefärbte Begriff „eurasisch“ (evrazijskij) gebraucht. 
Das Image Jelzins in der deutschen Öffentlichkeit hatte schon aufgrund der russischen Kriegsführung in Tschetschenien 1992-1996 gelitten. Seine Haltung in den Balkankriegen verschärfte das Image-Problem und bröckelte dann völlig wegen der auch für die russische Machtelite und die Öffentlichkeit peinlichen Auftritte des Präsidenten in offensichtlich angetrunkenem Zustand ab. Das allerdings sollte sich mit dem Amtsantritt seines von ihm und seiner „Familie“ ausgesuchten Nachfolgers ändern.

\section{Putin, „unser Mann in Moskau“}

Putins Amtsantritt verband sich in Teilen der Bundesregierung, politischen Parteien und der Öffentlichkeit mit der Idee, der neue Kreml-Chef hege aufgrund seiner deutschen Sprachkenntnisse und seiner Tätigkeit als KGBAgent in Dresden 1985-1990 ein besonderes Faible für Deutschland und werde sich deutschen Interessen gegenüber aufgeschlossen zeigen. ${ }^{18}$ Derartige Anschauungen erhielten bei seinem ersten Staatsbesuch in Deutschland und seiner Rede im Deutschen Bundestag im September 2001 enormen Auftrieb.

Putins (mit Ausnahme einer kurzen Einleitung) auf Deutsch gehaltene Rede war eine nicht anders als brillant zu bezeichnende Charakterisierung des postsowjetischen Russlands als ein europäischen Werten verpflichtetes Land, das den Weg einer nachhaltigen und unumkehrbaren Reformpolitik eingeschlagen habe. Im Gegensatz $\mathrm{zu}$ der in der Mittelfristigen Strategie vorgenommenen Zurückweisung gesamteuropäischer Integration und der Betonung der Andersartigkeit Russlands als „eurasische“ Macht versicherte er den Abgeordneten des Bundestages: „Russland ist ein freundlich gesinntes europäisches Land“ und „was die europäische Integration betrifft, so unterstützen wir nicht einfach nur diese Prozesse, sondern sehen sie mit Hoffnung.“

Apodiktisch erklärte Putin: „Das Hauptziel der Innenpolitik Russlands ist vor allem die Gewährleistung der demokratischen Rechte und der Freiheit, die Verbesserung des Lebensstandards und der Sicherheit des Volkes.“ Er bediente damit geschickt das nicht nur von Apologeten des Systems Putin und seiner Außenpolitik gezeigte Verständnis dafür, dass die Verwirklichung dieses Ziels schwierig sei und lange dauern könne. So beruhigte er kritische deutsche Gemüter mit den Worten: „Wir sind

18 „Unser Mann in Moskau“ ist der Titel eines Zeitschriftenartikels von Alexander Rahr (2004). Ähnlich der Untertitel seiner Putin-Biographie Der „Deutsche“ im Kreml (Rahr 2000). natürlich am Anfang des Aufbaus einer demokratischen Gesellschaft und einer Marktwirtschaft. Auf diesem Wege haben wir viele Hürden und Hindernisse zu überwinden. Aber abgesehen von den objektiven Problemen und trotz mancher - ganz aufrichtig und ehrlich gesagt - Ungeschicktheit schlägt unter allem das starke und lebendige Herz Russlands, welches für eine vollwertige Zusammenarbeit und Partnerschaft geöffnet ist.“

Putin zeigte sich infolgedessen „überzeugt“: „Wir schlagen heute eine neue Seite in der Geschichte unserer bilateralen Beziehungen auf und leisten damit unseren gemeinsamen Beitrag zum Aufbau des europäischen Hauses. " Die Glaubwürdigkeit seiner Überzeugung wurde dadurch erhöht, dass er von der Standardformel der „Befreiung“ Ostmitteleuropas abging und die geschichtliche Wahrheit der Errichtung eines sowjetischen Besatzungsregimes mit den Worten einräumte: „Wir [...] unterstützen europäische Integrationsprozesse als ein Volk, das gute Lehren aus dem Kalten Krieg und aus der verderblichen Okkupationsideologie gezogen hat.“19

Putins Rede wurde zwar von der Mehrheit der Abgeordneten mit einer stehenden Ovation gefeiert, aber nicht alle waren von der Aufrichtigkeit seiner Beteuerungen überzeugt. Im Grunde genommen spalteten sich Regierung, politische Parteien und Öffentlichkeit in unterschiedliche Lager mit unterschiedlichen Folgerungen und Ratschlägen hinsichtlich der gegenüber Russland zu verfolgenden Politik. An dieser Spaltung hat sich bis heute nichts geändert.

Eine Richtung von Interpretation und politischem Ansatz gegenüber Russland lässt sich als Apologie- und Appeasement-Schule bezeichnen, polemisch von ihren Kritikern als „Putin“- oder „Russland-Versteher“ benannt. Trotz aller in dieser Schule bestehenden Unterschiede gibt es doch wesentliche Übereinstimmungen..$^{20}$ Dazu gehörten die folgenden problematischen Interpretationsansätze:

- Die russische Innenpolitik wird als treibende Kraft für die Außenpolitik unterschätzt oder gar nicht in Rechnung gestellt. Letztere wird nicht als Teil einer innenpolitischen Dynamik, sondern als Reaktion auf äußere Bedrohungen interpretiert, deren Kern die von den Vereinigten Staaten dominierte NATO und ihre Osterweiterung sei.

- Die Interessen Russlands, auch seine Sicherheitsinteressen, werden mit den Interessen einer korrupten Machtelite gleichgesetzt, in der die Siloviki, also

19 Hervorhebung nicht im Original.

20 Eine ausführliche Darstellung und Kritik bei Adomeit 2019. 
führende ehemalige oder amtierende Mitglieder der Geheimdienste und Sicherheitsorgane, eine wichtige oder die beherrschende Rolle spielen.

- Die Wahrnehmungen, die „Russland“ zugeschrieben werden, werden nicht nach genuinen kognitiven Anschauungen Putins und der Siloviki einerseits und von ihnen instrumentell und propagandistisch genutzten Aspekten andererseits unterschieden.

- Russland wird nicht als eine revisionistische Macht betrachtet, die - auch mit der Anwendung militärischer Drohungen und dem Einsatz militärischen Gewalt - eine beherrschende Rolle im postsowjetischen Raum und verlorenen gegangenen Einfluss in der Welt wiedererlangen wolle, sondern als eine von begrenzten defensiven Sicherheitsinteressen geleitetes Land.

- Werte und Interessen sind radikal unterschiedliche Kategorien.

Derartige Anschauungen prägen die Anforderungen an die zu verfolgende Russlandpolitik. Dazu gehört der Ratschlag, von einer angeblich fehlgeleiteten Wertepolitik abzugehen und Interessenpolitik zu betreiben und dabei „Verständnis“ für russische Interessen zu zeigen und bereit zu sein, Kompromisse einzugehen. Eine „harte“ Linie, die darauf bestünde, dass Russland seine in der Charta von Paris und als Mitglied des Europarats und in anderen Verträgen eingegangenen Verpflichtungen ausführen solle, wird abgelehnt. Wirtschaftssanktionen seien „kontraproduktiv“, und auch nur symbolische und begrenzte militärische Maßnahmen wie Truppenstationierungen und Manöver in und mit östlichen NATO-Staaten sollten unterlassen werden, denn diese setzten einen „Rüstungswettlauf“ sowie eine gefährliche „Eskalationsspirale“ in Gang und beschwörten eine „akute[...] Kriegsgefahr“ in Europa herauf. $^{21}$

Eine auf derartigen Anschauungen beruhende Russlandpolitik hat die Bundesregierung gewissermaßen idealtypisch unter Kanzler Gerhard Schröder (1998-2005) verfolgt. Besorgnisse, dass Putin konsequent einen Kurs auf den Aufbau eines autoritären Systems betreibe und

21 Der Vorwurf, dass die Russlandpolitik der Bundesregierung dazu geführt habe, eine „akute Kriegsgefahr“ heraufzubeschwören, findet sich besonders deutlich bei Teltschik, vgl. Knobbe, Martin/Klaus Wiegrefe: Kohl-Berater Teltschik über Merkels Russlandpolitik: „Putin fühlt sich von den Europäern weggestoßen“, Spiegel Online, 8.3.2019; http://www.spiegel.de/politik/deutschland/angela-merkel-kohl-berater-horst-teltschik-kritisiert-russland-politik-a-1256918. html. Siehe auch Teltschik 2019, 7-8, 12, 224, Bahr 2018 (Beitrag Platzecks) und Platzeck 2020. dass dies ernste Auswirkungen auf die deutsch-russischen Beziehungen haben werde, wurden von ihm mit Bemerkungen vom Tisch gewischt, das Russland Putins sei eine „lupenreine Demokratie“, der russische Präsident betreibe eine „entschlossene Reformpolitik“ und im Land laufe „alles rechtsstaatlich“ ab. ${ }^{22}$

Dies wirft die Frage auf, welche politischen und gesellschaftlichen Kräfte in Deutschland im Großen und Ganzen derartige Anschauungen teilen und wie groß ihr Einfluss auf die Politik gegenüber Russland ist. Zuvorderst sind dabei die Haltung und Politik der SPD zu nennen. Ihre wichtigsten Führungspersönlichkeiten und Vorstandsmitglieder finden zwar manchmal kritische Worte über die Entwicklung der russischen Innen- und Außenpolitik, einschließlich der Annexion der Krim und militärischen Interventionen in Georgien, der Ostukraine und Syrien. Sie gehen aber keineswegs so weit wie Heiko Maas, der in seinem ersten Interview nach Übernahme des Außenministeriums Russland „zunehmend feindseliges“ Verhalten vorwarf. Es habe „erstmals seit Ende des Zweiten Weltkriegs mitten in Europa geächtete chemische Waffen eingesetzt." Cyberangriffe schienen zu einem integralen „Bestandteil russischer Außenpolitik“ zu werden.“ In einem so schwerwiegenden Konflikt wie in Syrien habe Russland den „Uno-Sicherheitsrat blockiert“ und in der Ukraine eine „Aggression“ verübt. Er hielt auch nichts von der Idee seiner SPD-Vorgänger, Sanktionen bereits abzubauen, wenn Russland nur einen Teil seiner Verpflichtungen aus dem Minsker Abkommen in der Ostukraine erfülle: „Es gibt klare Vereinbarungen, die vorsehen, dass Sanktionen erst abgebaut werden, wenn Russland seine Verpflichtungen erfüllt. Pacta sunt servanda.“23

22 Im Zusammenhang mit der Verhaftung des Vorsitzenden des Jukos-Ölkonzerns Michail Chodorkovskij sagte Schröder dass der Fall keine Ansatzpunkte dafür liefere, dass er „nicht rechtsstaatlich abgelaufen“ sei; Schröder stärkt Putin den Rücken: „Verstehe die Aufregung um Jukos nicht“, Frankfurter Allgemeine Zeitung, 9.7.2004.

23 Maas, Heiko: „Syrien ist nicht Auschwitz“ - Spiegel-Gespräch, Der Spiegel 16/2018. In letzter Zeit hat sich Gabriel allerdings von den Anschauungen der ehemaligen SPD-Führungstroika zu Russland entfernt. Dies zeigte sich unter anderem am 18.11.2019 in seinem Vortrag auf einer vom Deutsch-Russischen Forums e.V. und der Deutsch-Russischen Außenhandelskammer (AHK) organisierten Veranstaltung; siehe Sigmar Gabriel in Moskau: „Wir brauchen keine eigene deutsche Russlandpolitik“, Moskauer Deutsche Zeitung 19.11.2019; https:// mdz-moskau.eu/sigmar-gabriel-in-moskau-wir-brauchen-keineeigene-deutsche-russlandpolitik/. Gabriel wurde auch ausdrücklich von der (vernichtenden) Kritik Wolfgang Eichwedes an den im dritten „Aufruf“ erschienenen Beiträge ausgenommen (Eichwede 2019). Gabriel legte Anfang November 2019 sein Bundestagsmandat nieder und wechselte in die Wirtschaft. 
In Teilen seiner Partei und vor allem in den ostdeutschen Landesverbänden kamen diese klaren Worte offensichtlich nicht gut an. Bezeichnend dafür waren die nach einer Sitzung des Parteivorstands zur Außenpolitik von Generalsekretär Lars Klingbeil vorgebrachte Erinnerung daran, dass (als hätte Maas dies bestritten) die deutschrussischen Beziehungen für die SPD historisch und aktuell eine „sehr hohe Bedeutung“ hätten; Klingbeil mahnte: „Wir wollen den Dialog mit Russland, wir suchen den Dialog mit Russland, und wir wollen, dass der Dialog verstärkt wird.“24

Wie unterschiedlich von Führungsmitgliedern der SPD und den Landesverbänden die Akzente auch gesetzt sein mögen, mehrheitlich wird offensichtlich die Anschauung geteilt, dass die Ostpolitik Willy Brandts der Annäherung an die damalige Sowjetunion und die anderen Staaten des Warschauer Pakts auch heute noch oder wieder anwendbar sei und dass eine Neuauflage der Entspannungspolitik der 1970er-Jahre des letzten Jahrhunderts machbar sei. Auf dem Parteienspektrum gibt es auch in der CSU Unterstützung für die „weiche“, Putin-freundliche Linie. So gehört beispielsweise der ehemalige Parteivorsitzende und bayerische Ministerpräsident Edmund Stoiber zu den Initiatoren des zweiten von drei „Aufrufen“ an die Bundesregierung, eine grundsätzliche Neubewertung der Russlandpolitik vorzunehmen und eine neue Entspannungspolitik zu betreiben..$^{25}$ Zur Überraschung und entgegen Skepsis und Kritik aus der Partei schwenkte der FDP-Vorsitzende Christian Lindner - vermutlich aus wahltaktischen Gründen - in den Bundestagswahlen 2017 auf diese Linie ein. ${ }^{26}$ Ohne größere Vorbehalte und Verwässerungen teilen die AfD und Die Linke die Sicht des Kremls

24 SPD entschärft Streit über Russland-Politik und will Dialog, Frankfurter Neue Presse, 28.5.2018.

25 Schäfer, Helmut/Stoiber, Edmund/Teltschik, Horst/Verheugen, Günter/Vollmer, Antje: Konflikt mit Russland: Dialog statt Eskalation, Frankfurter Allgemeine Zeitung, 12.4.2018. Ein weiterer prominenter Kritiker der Russlandpolitik der Bundesregierung war Winfried Scharnagl, ehemaliges Mitglied des CSU-Parteivorstands und ehemaliger Chefredakteur des „Bayern-Kuriers“, desgleichen auch Peter Gauweiler, ehemaliger stellvertretender CSU-Vorsitzender, der einen der Beiträge in Bahr 2018 verfasst hat. Der erste, von mehr als 60 Persönlichkeiten aus Politik, Wirtschaft, Kultur und Medien unterzeichnete Appell, wurde im Dezember 2014 veröffentlicht, vgl. Herzog, Roman/Vollmer, Antje/Wenders, Wim/Schröder, Gerhard/u. a.: Ukraine-Krise: „Wieder Krieg in Europa? Nicht in unserem Namen!“ Die ZEIT vom 5.12.2014. Eine ausführliche Kritik der Appelle und der ihr zugrundeliegenden Vorstellungen hat der Autor an anderer Stelle vorgenommen: Adomeit 2019 und 2019a, siehe auch Eichwede 2019. 26 Wolfgang Kubicki, stellvertretender Bundesvorsitzender der FDP, MdB und Bundestagsvizepräsident, ist Verfasser einer der Beiträge im dritten „Aufruf“ (Bahr 2018). in außenpolitischen Fragen. Herausragende Beispiele dafür sind Besuche von Parteimitgliedern auf der Krim und ihre Teilnahme an von Moskau organisierten Wahlbeobachtungsmissionen auf der Halbinsel und in den Separatistengebieten der Ukraine (Doneck und Luhansk), Georgiens (Abchasien und Südossetien) und Moldova (Transnistrien), womit sie zur Legitimierung dieser international nicht anerkannten Gebiete beitragen. Mit Besuchen von AfD-Abgeordneten des Bundestags in Damaskus trägt die AfD auch zur Legitimierung des Assad-Regimes und der russischen Militärintervention in Syrien bei.

Welche Institutionen außerhalb des Parteienspektrums neigen aufgrund welcher Interessen ganz oder mehrheitlich der Verständnis-für-Russland-Schule zu? Zuvorderst ist der Ost-Ausschuss - Osteuropaverein der Deutschen Wirtschaft e. V. (OAOEV) zu nennen, der nach eigenen Angaben von sechs Spitzenverbänden der deutschen Wirtschaft getragen wird und rund 350 Mitgliedsunternehmen hat. ${ }^{27}$ Die prominenteste Putin-freundliche gesellschaftliche Organisation, die von sich behauptet, die „führende Stellung im deutsch-russischen Beziehungsgefüge" einzunehmen und in der auch die Wirtschaft eine wichtige Rolle spielt, ist das Deutsch-Russische Forum. ${ }^{28}$

Bemerkenswert bei der Haltung und dem Auftreten der Apologeten der Politik des Kremls über die Amtszeiten Putins hinweg ist eine Paradoxie: Die seit dem Krieg in Georgien kontinuierlich zunehmende Bereitschaft und die Fähigkeiten des Kremls, militärische Mittel einzusetzen und diese mithilfe der Militarisierung der Gesellschaft und Glorifizierung militärischer Erfolge in der russischen Geschichte zu legitimieren, haben die Vertreter der Verständnis-für-Russland-Schule nicht dazu bewogen, eine grundlegende Überprüfung ihrer Vorstellungen vorzunehmen, sondern ihre Kritik an der Russlandpolitik der Bundesregierung zu verschärfen. Stattdessen wird argumentiert, dass Reaktionen der NATO auf die russische Militärpolitik die Kriegsgefahr verschärften. Dies wirft die Frage auf, wie tragfähig die Russlandpolitik der Bundesregierung ist und welche Kräfte diese unterstützen.

27 Ost-Ausschuss $\neg$ - Osteuropaverein der Deutschen Wirtschaft e.V., https://www.oaoev.de/uber-uns.

28 Deutsch-Russisches Forum, https://www.deutsch-russischesforum.de. 


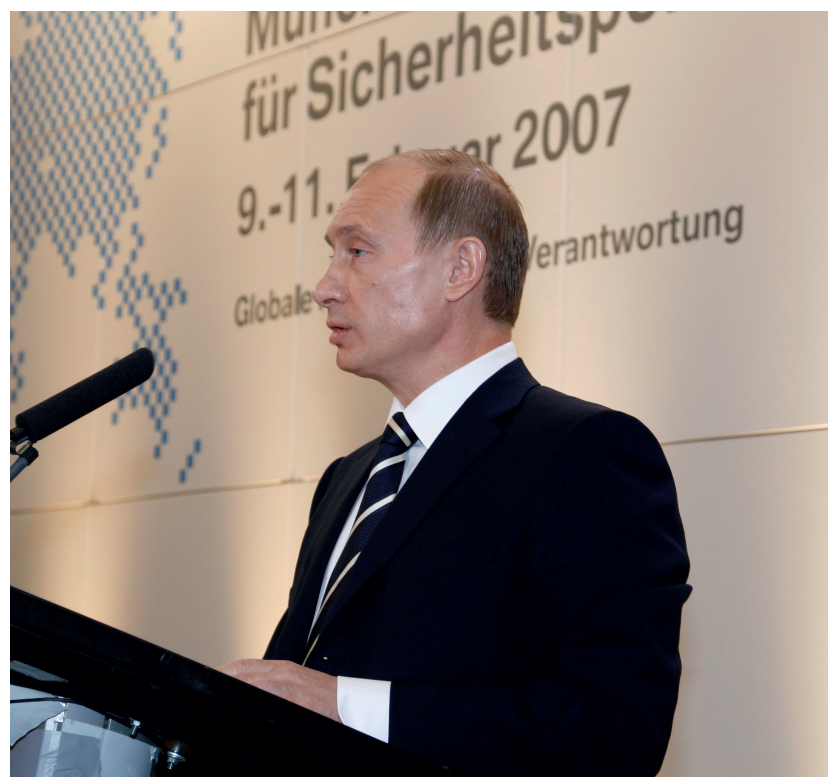

Putin kritisierte die NATO Erweiterung während der Münchener Sicherheitskonferenz 2007

\section{Träger der Russlandpolitik der Bundesregierung}

Im parteipolitischen Gefüge Deutschlands sind es mehrheitlich die Führungsgremien der CDU und des Bündnisses 90/Die Grünen, die eine realistische Bestandsaufnahme der Strukturelemente des Systems Putin und der diesem System entsprechenden Außenpolitik aufweisen. Man kann mit einiger Sicherheit davon ausgehen, dass die Bundeskanzlerin die Sicht Außenminister Maas‘ einer „zunehmend feindseligen“ Haltung des Kremls teilt. So führte sie am 13. Mai 2020 im Bundestag aus: „Es gibt eine Strategie Russlands der hybriden Kriegsführung. Das dürfen wir nicht verdrängen. Das ist Kriegsführung in Form von Cyber-Desorientierung und Desinformation. Das ist eine Strategie, kein Zufallsprodukt. Trotzdem bemühe ich mich um ein gutes diplomatisches Verhältnis zu Russland, aber so etwas macht es nicht einfach.“29

Derartige der Wirklichkeit entsprechende Bestandsaufnahmen der russischen Innen- und Außenpolitik teilen mit geringen Ausnahmen auch die Fachleute der

29 In diesem Zusammenhang wies sie auf den Hackerangriff auf ihr eigenes und andere Bundestagsbüros hin, der Ermittlungen des Generalbundesanwalts zufolge „harte Evidenzen“ für eine russische Beteiligung ergeben hätten. Dass die Spuren des Angriffs im Jahr 2015 zum russischen Geheimdienst führten, sei „ungeheuerlich“: https:// www.t-online.de/nachrichten/deutschland/id_87871608/angela-merkel-ueber-corona-politik-keinerlei-erhoehungen-von-steuern-.html.
Referate im Auswärtigen Amt, die sich mit Russland und den Ländern des postsowjetischen Raums befassen, ausgewiesene Experten für russische und osteuropäische Geschichte und Politik deutscher Universitäten und Forschungseinrichtungen, die Deutsche Gesellschaft für Osteuropakunde (DGO) mit der von ihr herausgegebenen Zeitschrift „Osteuropa“, die Leiter der politischen Stiftungen in Moskau, Kiyv oder anderen Hauptstädten im postsowjetischen Raum und frühere oder derzeitige Russland- und Osteuropakorrespondenten deutscher Medien.

Die Fachleute waren bereits frühzeitig skeptisch, ob der Amtsantritt Putins und sein besonderes Verhältnis zu Deutschland tatsächlich die deutsch-russischen Beziehungen aufblühen lassen würden. Ein herausragendes Beispiel dafür ist die Grundsatzrede Wolfgang Ischingers in seiner damaligen Eigenschaft als Staatsekretär im Auswärtigen Amt mit dem Titel „Was Berlin von Moskau erwartet und Moskau von Berlin erwarten kann“, die er im Juli 2000 an der Evangelischen Akademie Tutzing hielt. ${ }^{30}$ Ischinger wies auf Entwicklungen in Russland hin, die „Anlass zu großer Sorge“ gäben. Er nahm zur Kenntnis, dass Putin einen starken Staat aufbauen wolle, was seiner Ansicht nach allerdings verschiedene Dinge bedeuten könne. Der russische Staat könnte und sollte durch Demokratie und die Herrschaft des Rechts gestärkt werden nicht aber durch ein Mehr an Gewalt oder den Rückschritt zur Repression, wobei er auf Russlands ,unverhältnismäBigen Einsatz militärischer Gewalt, ohne klare Unterscheidung zwischen Kämpfern und Zivilisten“ sowie weitverbreitete Menschenrechtsverletzungen in Tschetschenien hinwies. Er erkannte zudem, dass es auch im Verhältnis $\mathrm{zu}$ Russland falsch sei, Werte und Interessen voneinander zu trennen: „Ohne Rechtssicherheit, ohne die strikte Respektierung und Durchsetzung bestehender Rechtsnormen [...] wird es kein wirkliches Vertrauen in die russische Wirtschaft geben. [...] Die mangelnde Rechtssicherheit in Russland ist ein wesentlicher Grund für die Kapitalflucht und die Zurückhaltung ausländischer Investoren. [...] Man kann die russische Regierung deshalb nur mit allem Nachdruck auffordern, den Ausbau des Rechtsstaats mit allem Nachdruck in Angriff zu nehmen." ${ }^{\text {31 }}$

Ischinger war sich zudem der engen Verbindung zwischen russischer Innen- und Außenpolitik bewusst. Putins Ziel, Russland wieder zu einer Großmacht zu machen, könne man zwar nicht kritisieren, meinte er. Er warnte aber, dass Größe im 21. Jahrhundert sich „nicht an der Zahl

30 Ischinger 2000.

$31 \mathrm{Zu}$ den systembedingten Grenzen für deutsch-russische wirtschaftliche Zusammenarbeit in Zahlen siehe Fußnote 9. 


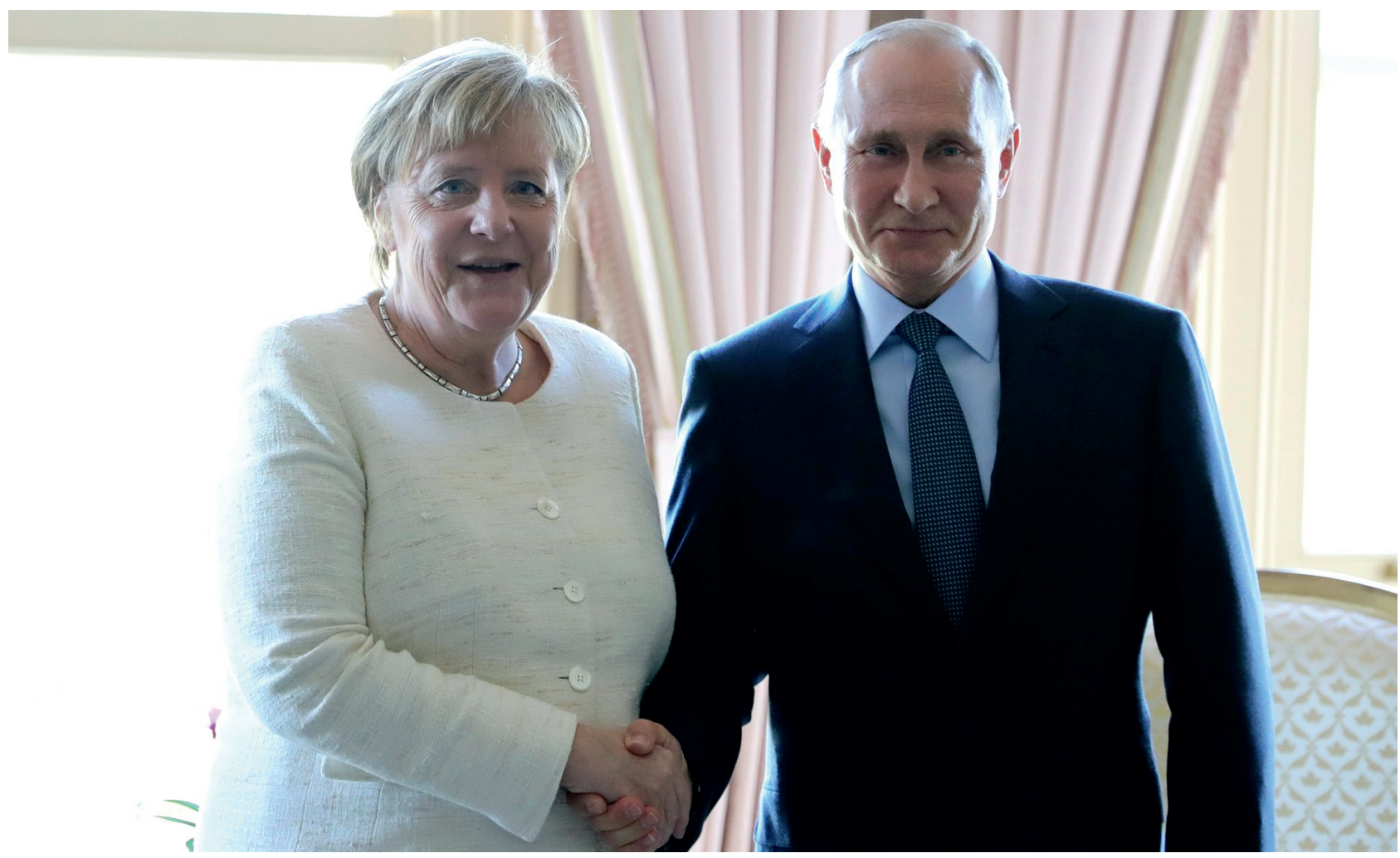

Bundeskanzlerin Merkel und Präsident Putin 2018

von Nuklearsprengköpfen oder Soldaten“ bemesse. Größe eines Landes beruhe vielmehr auf seiner „Wirtschaftskraft, Humankapital, einer dynamischen Gesellschaftsordnung und einem international attraktiven Bildungssystem. [...] Größe bemisst sich also heute nicht so sehr an Macht, sondern an der Kraft zu gestalten. Es ist wichtiger, zu überzeugen, als zu drohen, einzubinden, als zu beherrschen, es ist wichtiger, Partner zu gewinnen, als Gegner in Schach zu halten. Das sind die Gebote des 21. Jahrhunderts.“32

In diesem Zusammenhang übte Ischinger auch Kritik an Russlands Politik gegenüber seinen Nachbarstaaten: „Großmacht sein kann nicht gleichbedeutend sein mit einem Freibrief für mangelnde Rücksichtnahme auf kleinere Länder. Es werde „umso mehr Respekt und Verständnis für seine eigenen Interessen gewinnen, wie es die Interessen und Sensibilitäten seines Umfelds wahrzunehmen und in seinem eigenen Verhalten zu berücksichtigen versteht.“

Wie die Äußerungen der Bundeskanzlerin und ihres Außenministers zeigen, hat das Russland Putins die „Ge-

32 Ischingers „Gebote des 21. Jahrhunderts“ orientieren sich offensichtlich an den Thesen Joseph Nyes über die Bedeutung von Soft Power in der internationalen Politik. bote des 21. Jahrhunderts“ nicht befolgt. Allerdings gibt es eine Kluft zwischen den wirklichkeitsnahen Bestandsaufnahmen und der gegenüber Putins Russland tatsächlich verfolgten Politik. Dem Bewusstsein, dass der Kreml einen „zunehmend feindseligen“ und „aggressiven“ Kurs fährt, eine „Strategie der hybriden Kriegsführung“ und „Cyber-Desorientierung und Desinformation“ verfolgt sowie eklatante „Völkerrechtsverletzungen“ und „Kriegsverbrechen “ begeht, ${ }^{33}$ folgt keine Gegenstrategie, die darin bestünde, der russischen Führung hohe politische und wirtschaftliche Kosten aufzuerlegen.

So hat sich die Bundeskanzlerin - mit einer Ausnahme - immer auf den Standpunkt gestellt, dass die Nord-Stream-Ostseepipeline keine geopolitische oder strategische Bedeutung habe, sondern ein rein wirtschaftliches Projekt sei..$^{34}$ Norbert Röttgen (CDU), Vorsitzender des

33 Außenminister Maas am 27.2.2020 im VN-Sicherheitsrat zu den Bombenangriffen der russischen Luftwaffe in Syrien: „Willkürliche Angriffe auf die Zivilbevölkerung sind Kriegsverbrechen.“

34 Im April 2018 hatte Merkel eingeräumt, bei dem Projekt seien „natürlich auch politische Faktoren zu berücksichtigen“. Vermutlich spiegelt(e) diese Bemerkung ihre wirkliche Überzeugung wider. Die Kanzlerin nahm allerdings keine Kurskorrektur vor und kehrte zu der Standardformel vom „wirtschaftlichen Projekt“ zurück. 
Auswärtigen Ausschusses, hat wie Außenminister Maas die russischen Luftangriffe im Norden Syriens als „Kriegsverbrechen“ bezeichnet und das „Wegschauen“ und die Zurückhaltung des Westens als ,eine Schande“ und ,gegen unsere eigenen Sicherheitsinteressen" gerichtet und gefordert: „Wenn die Verbrechen weitergehen, muss es Sanktionen gegen Russland geben“. ${ }^{35}$ Die Bundesregierung hat dieser Forderung allerdings nicht entsprochen. Besonders kontrovers und schwierig durchzusetzen sind offensichtlich Maßnahmen, die Putins Kurs mit Kosten und militärische Risiken belegen. So war es innenpolitisch zwar möglich, Waffen an die Kurden im Irak zu liefern, aber noch nicht einmal eine Debatte zu führen, ob der Ukraine Waffen zur Verteidigung gegen die russische Aggression im Osten des Landes zur Verfügung gestellt werden sollten.

Die von Ischinger „mit großer Sorge“ im Jahr 2000 betrachteten Entwicklungstendenzen prägten sich in der Folgezeit voll aus und erreichten in der dritten Amtszeit Präsident Putins ihren Höhepunkt. Kritische Vorstufe dazu war das Scheitern der deutschen und EU-Neuauflage von Vorstellungen über die Modernisierung Russlands während der Amtszeit Medvedevs.

\section{Kollaps der Modernisierungs- partnerschaft}

Kurz vor dem Beginn der weltweiten Finanz- und Wirtschaftskrise 2008/2009 erklärte Putin: „Wenn wir den gegenwärtigen Pfad weiter beschreiten, werden wir nicht [...] in der Lage sein, die Sicherheit unseres Landes oder seine normale Entwicklung zu gewährleisten. Wir werden sogar unsere Existenz aufs Spiel setzen. ${ }^{36}$ Mitten in der Krise wiederholte Dmitrij Medvedev in seiner neuen Eigenschaft als Präsident die Warnung seines Vorgängers und Mentors: „Wir müssen Schlussfolgerungen aus den jüngsten Ereignissen ziehen. Solange die Ölpreise anstiegen, gaben sich viele der Illusion hin, dass strukturelle Reformen warten könnten. [...] Wir können aber nicht weiter herumtrödeln. Wir müssen mit der Modernisierung und der technologischen Aufbesserung unseres gesamten Industriesektors beginnen. Ich betrachte dies als eine

35 Röttgen fordert Sanktionen gegen Russland, FAZ.net, 18.2.2020. 36 Putin am 8.2.2008 in seiner Rede vor einer erweiterten Sitzung des Staatsrates der Russländischen Föderation, in der er seine „Entwicklungsstrategie Russlands bis 2020“ vorstellte; vgl. Putin, Vladimir: Vystuplenie na rasširennom zasedanii Gosudarstvennogo soveta „O strategii razvitija Rossii do 2020 goda“, http://kremlin.ru/events/ president/transcripts/24825, 8.2.2008.
Frage des Überlebens unseres Landes in der modernen Welt. “37

Medvedev kritisierte nicht nur die exzessive Abhängigkeit der russländischen Wirtschaft von hohen Ölpreisen, sondern auch die gesellschaftlichen und damit unausgesprochen die politischen Verhältnisse. Er beklagte die „Kultur des Rechtsnihilismus“, die in ihrem Zynismus auf dem europäischen Kontinent nicht ihresgleichen habe. Seine Landsleute müssten begreifen, dass, „wenn wir das halbsowjetische [...], archaische Gesellschaftssystem " und die ,jahrhundertealte Rückständigkeit“ überwinden und einen ,zivilisierten Staat aufbauen wollen, wir vor allem rechtsstaatliche" Verhältnisse einführen müssen.

Die Modernisierungskampagne, an deren Spitze sich Medvedev stellte, war dem Wort nach eindeutig. Seiner Kritik an den gesellschaftlichen Verhältnissen entsprechend sollte die Transformation der russländischen Wirtschaft zu einem modernen, innovativen und wettbewerbsfähigen Industriestaat mithilfe von Liberalisierung, Ermutigung privater Initiative, Abbau bürokratischer Hindernisse und Schutz von Unternehmern vor staatlicher Willkür bewerkstelligt werden.

Bedeutsam für die weitere Entwicklung des deutschrussischen Verhältnisses war die Tatsache, dass das Medvedev-Putin-Führungstandem die Modelle und Partner der Modernisierung von Wirtschaft und Gesellschaft im Westen suchte. Medvedev griff bereitwillig die von Außenminister Frank-Walter Steinmeier in seiner Rede in Ekaterinburg im Mai 2008 entwickelte Idee zur Gründung einer deutsch-russischen „Modernisierungspartnerschaft“ auf, die eine Konkretisierung der (immer noch offiziell im Sprachgebrauch der Bundesregierung bestehenden) „Strategischen Partnerschaft" darstellen sollte. ${ }^{38}$ Auch mit anderen europäischen Ländern und der EU vereinbarte Medvedev Modernisierungspartnerschaften. Aber nicht nur Europa sollte zur Modernisierung beitragen. Auch die USA waren dazu aufgerufen - eine Absicht, die er auf einer dreitägigen Amerikareise im Juni 2009 deutlich machte. So kündigte er nach dieser Reise an, dass sich die Errichtung seines Modernisierungsprojekts, des „Innovationszentrums“ in Skolkovo vor den Toren Moskaus, „am Modell Silicon Valley und anderen ausländischen Zentren ausrichten" solle. ${ }^{39}$

37 Medvedev, Dmitrij (2009): Poslanie Federal'nomu Sobraniju Rossijskoj Federacii, http://kremlin.ru/transcripts/5979, 12.November 2009.

38 Einzelheiten dazu bei Adomeit 2011.

39 Medvedev, Dmitrij (2009): Poslanie Federal'nomu Sobraniju Rossijskoj Federacii, http://kremlin.ru/transcripts/5979, 12.November 2009. 
Wie so viele Kampagnen in der Sowjetära verpuffte auch diese im neuen Russland - mit ernsten Konsequenzen für das deutsch-russische Verhältnis. Der Grund dafür lag im innenpolitischen Machtkalkül des Kremls. Die radikale Kritik Medvedevs an den wirtschaftlichen und gesellschaftlichen Verhältnissen, insbesondere an der systemimmanenten Korruption staatlicher Organe und ihrer Verfilzung mit der organisierten Kriminalität, hatte breite Resonanz gefunden. Die Umfragewerte der Kreml-nahen Partei „Einiges Russland“ waren in den vorausgegangenen Monaten stark abgesackt - nicht zuletzt wegen der vom Oppositionspolitiker, Blogger und Chef der Nichtregierungsorganisation „Fonds zur Korruptionsbekämpfung“, Aleksej Naval'nyj, in Umlauf gebrachten Brandmarkung der Partei als „Partei der Diebe und Gauner“. Auch die Popularitätswerte für Putin waren stark zurückgegangen.

In den Wahlen zum Parlament am 4. Dezember 2011 und zum Präsidentenamt am 4. März 2012 entlud sich die Unzufriedenheit. Die Regierungspartei erlitt schwere Verluste und auch Putins Ergebnisse in den Präsidentschaftswahlen blieben hinter den Werten zurück, die er vor vier Jahren erzielt hatte.

Das Bedeutsame an den Wahlen waren allerdings nicht die Ergebnisse selbst, sondern die Massendemonstrationen gegen Wahlfälschungen und Wahlmanipulationen. Dagegen hatten Zehntausende von Menschen in Demonstrationen protestiert und auf Plakaten ein „Russland ohne Putin“ gefordert. Für den Kreml problematisch war vor allem die Tatsache, dass die Demonstranten zu einem großen Teil aus einer im Entstehen begriffenen Zivilgesellschaft stammten - aus Mitgliedern international vernetzter russischer NGOs, gut ausgebildeten Fachkräften und erfolgreichen Unternehmern der Privatwirtschaft. Aus der Sicht der Moskauer Machtelite hatte der angeblich von westlichen Regierungen mittels ihrer Geheimdienste und sogenannten NGOs auf dem Balkan und in Ostmitteleuropa verbreitete Virus der „Farbrevolutionen“ begonnen, auf Russland überzuschwappen.

Die Folgerungen, welche der Kreml aus diesen Befürchtungen zog, waren eindeutig. Den Gefahren musste im Lande selbst und in den Nachbarstaaten energisch begegnet werden. Entsprechend verschwand mit Beginn der dritten Amtszeit Putins als Präsident nunmehr für sechs statt wie vorher für vier Jahre - der Begriff der „Modernisierung“ praktisch völlig aus dem offiziellen Diskurs. Wenn er gebraucht wurde, dann fast ausschließlich im Zusammenhang mit der Modernisierung der russischen Streitkräfte. Per Gesetz wurden russische Vereine und Menschenrechtsgruppen, sofern sie sich politisch betätigten und Spendengelder aus dem Ausland erhielten, unter Androhung schwerer Strafen verpflichtet, sich in einem besonderen Register des Justizministeriums als ,ausländische Agenten“ eintragen zu lassen. Verschiedene in Russland tätige ausländische und internationale Nichtregierungsorganisationen wurden $\mathrm{zu}$,unerwünschten Organisationen“" erklärt und ihre Tätigkeiten verboten. Der Kreml verabschiedete sich von der Möglichkeit, eine breit angelegte Reformpolitik mithilfe europäischer und amerikanischer Investitionen durchzuführen, und er ging zu einem groß angelegten Bemühen über, die angeschlagene Legitimität auf national-patriotischer und scharf anti-westlicher Basis wiederherzustellen.

\section{Fazit}

Wie das Scheitern der deutsch-russischen und EU-Modernisierungspartnerschaften zeigt, sind die Entfremdung und die tiefe Krise zwischen Berlin und Moskau an inneren Faktoren der Entwicklung in Russland festzumachen. Diese haben das russische Außenverhalten bestimmt. In dem Maße, in dem äußere Faktoren die Außenpolitik des Kremls und damit auch seine Politik gegenüber Deutschland beeinflussen, ist es im Wesentlichen die Sorge der russischen Machtelite, dass das ordnungspolitische Vorbild und die sozioökonomische Attraktivität des Westens eine Bedrohung der Legitimität ihrer Herrschaft in Russland darstellt und ihren Einfluss in der von ihr deklarierten Interessensphäre untergräbt. Dies bietet eine der wesentlichen Erklärungen dafür, warum der Kreml zwei umfassende Kampagnen führt: eine nach innen gerichtete, welche die USA und die europäischen Länder (einschließlich Deutschlands) als von unüberbrückbaren sozialen und politischen Gegensätze zerrissen darstellt, und eine zweite, im Ausland geführte, die mit „aktiven Maßnahmen“ darauf Einfluss nehmen will, die Wirklichkeit diesem Zerrbild anzupassen.

Konkret beinhaltet die nach innen gerichtete Kampagne die Ablehnung westlicher Vorbilder von Demokratie und Rechtsstaat zugunsten eines eigenständigen russischen Entwicklungsweges, Ausgrenzung regierungsunabhängiger, regimekritischer Organisationen mit Verbindungen zum Ausland als staatsgefährdend und Verbote missliebiger ausländischer und internationaler Organisationen.

Maßnahmen dieser Art tragen dazu bei, vor allem in Deutschland gängigen Ansätzen, mithilfe von Verbindungen und Verflechtungen mit der russischen Zivilgesellschaft Spillover-Effekte auf die Politik des Kremls zu bewirken, den Boden zu entziehen. Derartige Bemühungen um 
Einflussnahme werden vom Kreml als „Einmischung in die inneren Angelegenheiten“ des Landes gebrandmarkt.

Auch in dieser Hinsicht trifft Putins Charakterisierung zu: „Die Sowjetunion - auch das ist Russland, nur unter einem anderen Namen." ${ }^{40}$ Die Herausforderung für das Sowjetsystem in der UdSSR und im europäischen Teil seines Herrschaftsbereichs war nicht ein von der NATO inszenierter militärischer Rollback, sondern systempolitischer „Revisionismus“ und westliche Entspannungspolitik. Im Bewusstsein der Sowjetführer war eine entspannungsfreundliche SPD für das Sowjetimperium „gefährlicher“ als eine auf „Positionen der Stärke“ beharrende CDU/CSU. Entsprechend dienten die Militärinterventionen in Ungarn 1956 und in der Tschechoslowakei 1968 sowie die Drohungen mit militärischem Eingreifen in Polen 1956 und 1980-1981 nicht, wie die sowjetische Propaganda behauptete, der Abwehr einer äußeren Gefahr, sondern dazu, einen systempolitischen „Frühling“ Prager oder jeglicher anderer Prägung auszuschalten. Das Russland Putins ist zu diesem Verhaltensmuster der Sowjetunion Brežnevs zurückgekehrt.

Deutsche Russlandpolitik muss infolgedessen ein Paradox zur Kenntnis nehmen. Wie in der Sowjetära schaffen westliches Entspannungsbemühen sowie Kompromissund Kooperationsbereitschaft bei der gegenwärtigen Verfassung des Systems Putins und seiner darauf beruhenden Außenpolitik nicht die Voraussetzungen für eine nachhaltige Verbesserung der Beziehungen, sondern behindern diese. Der Grund dafür liegt darin, dass der Kreml Putins strategische Gegnerschaft zum Westen nicht als Kollateralschaden seiner Politik in Kauf nimmt, sondern gezielt als Mittel für Legitimierung des Herrschaftssystems einsetzt.

Teil dieser Strategie ist der verstärkte Einsatz militärischer Instrumente in der Außenpolitik, wie er in der Militärintervention über Südossetien hinaus nach Abchasien und ins Kernland nach Georgien, der Annexion der Krim und den militärischen Interventionen in der Ostukraine und in Syrien seinen Ausdruck gefunden hat. Innenpolitisch wird dieser Kurs durch die Glorifizierung vergangener militärischer Siege und die angebliche Notwendigkeit gerechtfertigt, westlichem Druck zu widerstehen.

Was also sind die Voraussetzungen dafür, dass die Krise in den deutsch-russischen Beziehungen überwunden und eine nachhaltige Verbesserung erzielt werden kann? Notwendig wäre neues politisches Denken in Moskau beziehungsweise die Rückkehr zum Neuen Politischen Denken Michail Gorbačëvs. Dazu würden die folgenden miteinander verbundenen Strukturelemente gehören:
- der Abbau des Bildes vom Westen als Feind, der immer und ewig darauf aus sei, Russland in die Knie zu zwingen;

- die Abkehr von einer Politik, die sich von Exporten von Öl, Gas und Waffen abhängig macht und militärische Macht als wichtigstes Instrument der russischen Außenpolitik einsetzt;

- die Abwendung von Gedanken und Gesetzesinitiativen über den Vorrang russischen Rechts und den Weg zurück zur Anerkennung und Anwendung universeller Werte;

- in den Beziehungen zu den Nachbarstaaten die Abkehr vom Prinzip der begrenzten Souveränität, imperialen Kontrollbefugnissen und der Praxis verdeckter oder offener militärischer Intervention zurück zum Prinzip der Freiheit der Wahl;

- in der inneren Verfassung des Landes die Abkehr von autoritären, monolithischen und zentralistischen Ordnungsprinzipien sowie von Konzepten wie „souveräner“ und „gelenkter“ Demokratie zurück zu Demokratizacija, zur Zielsetzung des Aufbaus einer Demokratie ohne einschränkende Adjektive mit einer funktionierenden Gewaltenteilung und rechtsstaatlichen Verhältnissen;

- $\quad$ in der Wirtschaft die Überwindung der Abhängigkeit von Rohstoffen sowie die Beseitigung der Vorrangstellung des militärisch-industriellen Komplexes, der Verfilzung der Staatsbürokratie mit der organisierten Kriminalität und der Einschränkung freien Unternehmertums zurück zur Perestrojka, zum Umbau des Landes zu Marktwirtschaft mit fairem Wettbewerb;

- im Beziehungsgeflecht zwischen Staat und Gesellschaft die Aufgabe der Gleichschaltung von Medien und Meinungen, der alles durchdringenden staatlichen Propaganda und der Gängelung einer aktiven Zivilgesellschaft zurück zu Glasnost, zu Offenheit und Öffnung der Gesellschaft.

Ein derartiger Politikwandel wird nur zu erreichen sein, wenn gerade die deutsche Politik mit großer Einheitlichkeit diese Forderungen immer wieder an die russische Führung richtet. Auf die Stimme Berlins wird in Moskau gehört. Solange in SPD, CSU, AfD und Linkspartei die Stimmen derjenigen überwiegen, die ein Nachgeben des Westens gegenüber angeblich berechtigten Großmachtinteressen Russlands verlangen und die die innenpolitische Bedingtheit der russischen Außen- und Militärpolitik ignorieren, wird dieser Effekt aber nicht eintreten. 


\section{Literatur}

Adomeit, Hannes/Bomsdorf, Falk (1990): Das „neue Denken“: Grundzüge und Verwirklichung, in: Adomeit, Hannes/ Höhmann, Hans-Hermann/Wagenlehner, Günter (Hrsg.): Die Sowjetunion unter Gorbatschow. Stuttgart: Kohlhammer Verlag, 261-296

Adomeit, Hannes (1994): Russia: Partner or Risk Factor in European Security? London: International Institute for Strategic Studies (Adelphi Paper 285)

Adomeit, Hannes (2011): Russlands Modernisierungspartnerschaften: Ursprünge, Inhalte und Erfolgsaussichten, in: Russland modernisiert sich - oder doch nicht? Sozialwissenschaftliche Schriftenreihe des Internationalen Instituts für Liberale Politik (IILP), (36-37), 25-72

Adomeit, Hannes (2013): Partner oder Störfaktor - Russlands Rolle in der europäischen Nachbarschaft, in: Stratenschulte, Eckart D. (Hrsg.): Grenzen der Integration. Europas strategische Ansätze für die Nachbarregionen. Baden-Baden: Nomos, 121-151

Adomeit, Hannes (2019): Müssen wir Russland besser verstehen lernen? Eine kritische Auseinandersetzung mit den Argumenten für eine neue Russlandpolitik, Sirius - Zeitschrift für strategische Analysen, 3 (3): 224-241
Alexandrova, Olga (1997): Der außenpolitische Konsens in Russland. Köln: Bundesinstitut für ostwissenschaftliche und internationale Studien (Aktuelle Analysen 17)

Bahr, Adelheid, Hrsg. (2018): Warum wir Frieden und Freundschaft mit Russland brauchen. Ein Aufruf an alle von Matthias Platzeck, Peter Gauweiler, Antje Vollmer, Oskar Lafontaine, Gabriele Krone-Schmalz, Peter Brandt, Daniela Dahn und vielen anderen, Frankfurt: Westend Verlag

Eichwede, Wolfgang (2019): Gefährliche Ratgeber. [Kritik an Bahr 2018] https://libmod.de/wolfgang-eichwede-gefaehrlicheratgeber/.

Ischinger, Wolfgang (2000): Die Zukunft Russlands liegt in Europa. Was Berlin von Moskau erwartet und Moskau von Berlin erwarten kann. Frankfurter Allgemeine Zeitung, 11. Juli (3)

Platzeck, Matthias (2020): Wir brauchen eine neue OstpolitikRussland als Partner. Berlin: Propyläen.

Rahr, Alexander (2000): Wladimir Putin: Der „Deutsche“ im Kreml. München: Universitas

Rahr, Alexander (2004): Unser Mann in Moskau, Cicero (11), 28-35

Simon, Gerhard (1997): Der patriotische Konsens in Russland. Köln: Bundesinstitut für ostwissenschaftliche und internationale Studien (Aktuelle Analysen 11)

Teltschik, Horst (2019): Russisches Roulette. Vom Kalten Krieg zum Kalten Frieden. München: C.H. Beck 